\title{
21
}

\section{ESTIMATING QoS PARAMETERS FOR ATM TRAFFIC USING ITS ENTROPY}

\author{
N.G.Duffield ${ }^{1}$, J.T.Lewis ${ }^{2,3}$, Neil O'Connell ${ }^{2}$, \\ Raymond Russell ${ }^{2}$ and F.Toomey ${ }^{2}$
}

\section{Abstract}

How will ATM carriers allocate the band-width required to guarantee the quality-ofservice promised in their customer contracts? How can customers exploit to their advantage the tariff structures offered by the carriers? Both carrier and customer will need to measure QoS (quality-of-service) parameters.

Existing proposals involve modelling: fitting a statistical model to the input traffic and calculating QoS parameters from the model. Doubts have been expressed about this procedure because data traffic is bursty and cannot be described by a model with a small number of adjustable parameters. In any case, a statistical model of traffic contains more information than we need to calculate the QoS parameters. Our approach is more radical: we estimate directly the thermodynamic entropy of the data-stream at an input-port; from this, the QoS parameters can be calculated rapidly. We give a brief exposition of our strategy and then we describe some of the algorithms we have devised to implement it.

\section{The Problem}

Streams of incoming cells are multiplexed and stored in a buffer which is emptied at a constant service-rate. If the buffer is too small, cells may be lost by overflow; if the buffer is too large, long queues may develop and some cells may experience unacceptably long delays.

The ATM forum has proposed the use of three parameters to measure the quality of service experienced by traffic as it passes through a queueing system. These are:

- the cell loss ratio (ratio of cells lost to total number of cells);

\footnotetext{
${ }^{1}$ School of Mathematical Sciences, Dublin City University, Dublin 9, Ireland

${ }^{2}$ Dublin Institute for Advanced Studies 10 Burlington Road, Dublin, Ireland

${ }^{3}$ Author presenting paper
} 
- the mean cell delay;

- the cell delay variance ('jitter').

The problem is to find safe bounds on the queueing contribution to these parameters. In Appendix A, we show that safe bounds on all three QoS parameters can be obtained from a bound on the tail of the queue-length distribution. Fundamental to all work on estimating QoS parameters is the following result from the theory of large deviations:

if we know the rate-function of the arrivals process, we can calculate a bound on the tail of the queue-length distribution.

The problem is thus reduced to that of determining the rate-function of the arrivals process (the number of cells entering the buffer in each clock-cycle). The current practice is to model the arrivals process:

- choose a statistical model;

- fit the model to the traffic (using moments, for example);

- compute the rate-function for the model;

- compute the bound on the queue-length distribution;

- estimate the QoS parameters.

There are practical difficulties associated with this programme which hinder its implementation:

- it is difficult to automate the selection of a model;

- bursty traffic cannot be modelled using a small number of parameters;

- the estimation cannot be performed in real time.

There is also an objection in principle to this programme:

- it wastes resources - a good model contains more information about the arrivals process than is required for the estimation of the QoS parameters.

These considerations have triggered the search for alternatives to modelling. A full characterisation of the traffic is not required in order to compute a bound on the queue-length distribution; we saw that all we need is the rate-function of the arrivals process, so why not estimate the rate-function directly from the data, by-passing the modelling? There are good reasons for believing that this might work. Since the work of Ruelle and Lanford, it has been known that the rate-function of large-deviation theory is the same kind of mathematical object as the entropy-function of equilibrium thermodynamics. The ratefunction and the entropy-function have this in common: they encapsulate concisely the 
relevant information about the system. For an ideal gas, the entropy-function can be calculated from first principles; for a real gas, one could choose a statistical model, fit the the parameters of the model to measured properties of the gas - virial coefficients, for example, compute the entropy-function from the model and use the entropy-function to compute the bulk properties of the gas. This is not the practice of chemical engineers: they measure the entropy-function or use the tables of measured values available in the literature.

Our claim is this:

for the purposes of estimating QoS parameters, it is enough to know the ratefunction of the ATM traffic stream; the modelling procedure can be by-passed if we can estimate the rate-function directly.

It turns out that it is better to estimate a transform of the rate-function, the scaled cumulant generating function (CGF), rather than the rate-function itself.

\section{Estimating the Scaled CGF}

We give a simple sketch of the theory of the statistical estimation of the scaled CGF and its use in estimating QoS parameters; further details are given in Appendix C. The scaled $C G F$ (cumulant generating function) $\lambda$ of the arrivals process is defined by

$\lambda(\theta):=\lim _{n \rightarrow \infty} \frac{1}{n} \log E \exp \left(\theta \sum_{i=1}^{n} X_{i}\right)$.

where $X_{i}$ is the number of cells arriving in the $i$-th clock-cycle. Provided this limit function exists ( and satisfies some technical conditions ), the rate-function $I(w)$ exists and is determined by it; the asymptotic slope $-\delta$ of the logarithm of the queue-length distribution can be calculated directly from $\lambda$ using the formula

$\delta=\max \{\theta: \lambda(\theta) \leq s \theta\}$.

The scaled CGF exists for a wide class of stationary processes. If we are to estimate $\lambda$ empirically, it is important that the arrivals process be approximately stationary; provided this is the case, and the process is mixing in the sense that there exists a block-size $K$ such that the block-sums

$\tilde{X}_{1}:=\sum_{i=1}^{K} X_{i}, \quad \tilde{X}_{2}:=\sum_{i=K+1}^{2 K} X_{i}, \ldots$

are approximately independent and identically distributed, we can estimate $\lambda$ using

$\hat{\lambda}(\theta):=\frac{1}{K} \log \frac{1}{N} \sum_{i=1}^{N} e^{\theta \bar{X}_{i}}$ 
and $\delta$ using

$\widehat{\delta}:=\max \{\theta: \hat{\lambda}(\theta) \leq s \theta\}$.

The advantage of this estimator is that it makes no assumptions about the data other than that it is stationary and mixing. The disadvantage of $\hat{\lambda}$ is that it involves an inevitable trade-off when choosing the block size $K: K$ must be chosen sufficiently large that $\tilde{X}_{i}$ and $\tilde{X}_{i+1}$ are approximately uncorrelated, but not so large that the number of observations $N$ of $\tilde{X}$ is too small. It can be shown (via the correlation inequality) that the effect of positive correlations between $\tilde{X}_{i}$ and $\tilde{X}_{i+1}$ is to make $\hat{\lambda}$ a consistent underestimator of the true CGF $\lambda$. Thus, as the block size $K$ increases, $\hat{\lambda}(\theta)$ typically increases at first towards the limiting value $\lambda(\theta)$, but at large $K$ starts to 'wobble' with the effect of statistical noise. We have not yet resolved all the issues involved in choosing an optimal block size $K$. The procedure adopted at present is to maximise $\hat{\lambda}$ over a range of values for $K$ and then resolve empirically the question of whether the estimator converges to the true value of $\lambda$ before the onset of noise.

\section{Testing the Estimator}

If a stream of data with CGF $\lambda(\theta)$ is passed into a single-server queue with service rate $s$ cells per time-slot, then results from the theory of large deviations tell us that the queue-length distribution $\mathbb{P}(Q>b)$ is asymptotically log-linear in $b$ : for $b$ large,

$\log \mathbb{P}(Q>b) \rightarrow-\delta b+c$,

where the slope $\delta$ is given by

$\delta=\max \{\theta>0: \lambda(\theta)<\theta s\}$.

This suggests a simple method of testing the estimator $\hat{\lambda}$. We pass the data through a queue and then compare by eye the graph of $\log \mathbb{P}(Q>b)$ with the straight line of slope

$\hat{\delta}=\max \{\theta>0: \hat{\lambda}(\theta)<\theta s\}$.

Note that $\hat{\delta}$ is just the ordinate of the intercept of the graph of $\hat{\lambda}$ with the line of slope $s$ through the origin. The following properties of $\hat{\lambda}$, which hold quite generally, are important considerations when estimating $\delta$ :

- $\hat{\lambda}$ is convex (the graph always lies below the chord);

- $\hat{\lambda}(0)=0$;

- $\hat{\lambda}^{\prime}(0)$ is the mean activity;

- for $\theta$ large, the largest observation $\tilde{X}_{\max }$ of $\tilde{X}$ dominates the arithmetic mean in (4), so that asymptotically $\hat{\lambda}$ is a straight line with slope $\tilde{X}_{\max } / K$. 


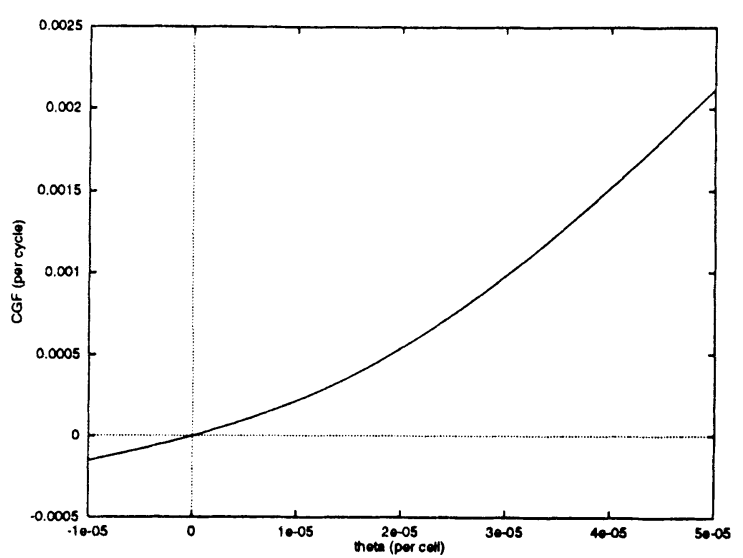

Figure 1: Graph of a typical CGF

The typical form of the graph of $\hat{\lambda}$ is shown in figure 1 . We see that as $s$ increases the intercept moves to larger and larger $\theta$-values, so by varying $s$ we can test the accuracy of the estimator $\hat{\lambda}(\theta)$ over a range of values of $\theta$. However, if $s$ is smaller than the mean activity (and hence the queue is unstable), there is no intercept and no value for $\hat{\delta}$ is obtained. Nor is there any intercept when $s$ is larger than $\tilde{X}_{\max } / K$ : in this case the estimator sees a service capacity that is always greater than the arrivals, so that the queue is always empty (but this may not really be the case; keep in mind that $\tilde{X}_{\max } / K$ represents the arrivals averaged over a block of length $K$ cycles, and is typically a decreasing function of $K$ ). In general, there is a range of service rates starting at the mean activity and ending at $\tilde{X}_{\max } / K$ for which results may be obtained, and a sub-range of this for which the results are reliable. The size of these ranges is expected to depend on the statistical properties of the traffic data.

Note that since $\hat{\lambda}(\theta)$ tends to under-estimate $\lambda(\theta)$ for all $\theta$, we expect $\hat{\delta}$ to over-estimate $\delta$ with the size of the error decreasing as the block size is increased.

\section{Results for the Fairisle Data}

The 'Fairisle data' consists of twelve sets of ATM traffic measurements taken on the Fairisle network at the University of Cambridge by Simon Crosby. Each set contains a list of successive cell inter-arrival times measured at a fixed point in the network during the transfer of data by ftp. The inter-arrival times are expressed in multiples of the system clock period which was set to 3.45 microseconds. We applied the procedure outlined above to each of the data sets and will describe here the results for one of them, named 'ftpfast.data'. As far as estimating the accuracy of $\hat{\lambda}$ is concerned, the results for the other sets were similar. The data in this sets was measured at the input to the network, before multiplexing with other traffic.

Prior to analysis the data was converted into 'cells-per-unit-time' format, and aggregated to an initial block size of 3.45 milliseconds. Hereafter, when we mention the term 
'clock-cycle', we are referring to this time unit. The activity measurements were used to calculate $\hat{\lambda}$ and $\hat{\delta}$ for a range of block sizes $K$ and service rates $s$. Measured values of $\hat{\delta}$ were compared with the slope of 'watermark' plots of $\log \mathbb{P}(Q>b)$ against $b$, produced by passing the activity data through a virtual queue and counting the number of times the queue length exceeds each 'watermark' $b$.

Figure 2 is an activity plot for the data set ftp-fast.data. Note the sharp level shift at $t \approx 90$ seconds. The effect of this shift is that the queue-length distribution for high service rates is determined almost entirely by the last 20 seconds of data; the contribution from this region also dominates in the calculation of $\hat{\lambda}$.

The minimal value of $\hat{\delta}$ occurs at block size $K=1624$ clock-cycles ( 5.6 seconds) and service rate $s=18.5$ cells per clock cycle. Figure 3 is a watermark plot computed for this service rate together with a straight line of slope $-\hat{\delta}$ (plotted through the origin). The fall-off of the watermark at large values of $b$ is caused by lack of data. Clearly, the graph shows a good match between $\hat{\delta}$ and the watermark slope. The average activity of the data is about 17.8 cells per clock cycle, so here we are dealing with a service rate just above the critical value for stability.

At service rates high compared to the critical rate, much poorer results were obtained with $\hat{\delta}$ over-estimating the watermark slope considerably. This lack of accuracy is caused by the small sample size at this aggregation level which has a more severe effect on the estimates at high service rates. Choosing an inappropriately low block size also leads to over-estimates of $\delta$.

The analysis of the other data sets replicated these results. For near-critical service rates, accurate estimates were obtained provided the block-size used was large (of the order of one second). For higher service rates, results were poor.

Figures 4-6 depict the QoS parameter bounds obtained for the traffic set ftp-fast.data. The value of $\mu$ was obtained by observing the frequency of overflow for a buffer of size one cell. To estimate $\delta$ accurately, we used a low service rate (one close to the critical rate required to make the queue stable ). It's worth noting that, at higher service rates, the quality of service experienced by the traffic is guaranteed to be better than that at the critical rate, so our results represent absolute 'worst case' bounds.

\section{Conclusions and Further Work}

Our results for the Fairisle data show that the simple CGF estimator (4) works well for small values of $\theta$ but poorly at larger values. This leads to good performance when predicting the watermark slope $\delta$ for low service rates but poor performance when attempting prediction for high rates. The reason for this behaviour is fairly clear; it has its origin in the necessity of using large block sizes to remove bias due to correlations between blocks. At these large block sizes the high end of the distribution of the aggregated arrivals $\tilde{X}$ is difficult to observe accurately, or even at all. But it is precisely this end of the distribution which contributes predominantly to $\lambda(\theta)$ when $\theta$ is large. Thus the value of $\hat{\lambda}(\theta)$ is 
inaccurate in this region. On the other hand using low block sizes leads to over-estimates of $\delta$ because $\hat{\lambda}$ is highly susceptible to bias.

The obvious way to remedy these problems is to develop a better estimator. For example, an estimator that is robust in the face of small correlations between blocks would allow us to use a smaller block size and thus reduce the amount of data needed to accurately determine the distribution of $\tilde{X}$. Since the estimator we are using at present takes no advantage at all of any known properties of the data it seems likely that much progress can be made in this direction.

Another possible approach is to look at ways of estimating the high end of the distribution of $\tilde{X}$ when it cannot be observed directly. This could be done for example by making some simple and general assumptions about the frequency of large bursts in the traffic. Note that an exponential bound on the frequency of such bursts follows immediately from the queue-length formula $(6)$; if we let $\tilde{X}_{K}$ be the arrivals and $W_{K}$ be the workload in a block of size $K\left(W_{K}=X_{K}-K s\right)$ then

$$
\begin{aligned}
\mathbb{P}\left(\tilde{X}_{K}>x\right) & =\mathbb{P}\left(W_{K}>x-K s\right) \\
& \leq \mathbb{P}\left(\sup _{t} W_{t}>x-K s\right) \\
& =\mathbb{P}(Q>x-K s) \\
& \approx e^{-\delta x-\delta K s} .
\end{aligned}
$$

Making use of this and any other known properties of the distribution of $\tilde{X}_{K}$ will assuredly lead to improvements in the performance of the estimator.

\section{Notes}

Several proofs of formula (7) have appeared in the literature: a heuristic argument can be found in Kesidis, Walrand and Chang [5] and proofs under very general conditions in Glynn and Whitt [4] and in Duffield and O'Connell [3]; further bibliographical details can be found in de Veciana, Courcoubetis and Walrand [10].

The connection between the theory of large deviations and thermodynamics was pointed out by Ruelle [8] and Lanford [6] over twenty-five years ago but only now is it beginning to be exploited; it is explained in a recent paper by Lewis and Pfister[7]. An account of related ideas addressed to a general audience has been given by Ruelle [9].

Acknowledgements This work was supported by grants from EOLAS and Mentec Computer Systems Ltd. under the Higher Education -Industry Cooperation Scheme. We thank Simon Crosby and Ian Leslie for making the ATM traffic measurements available to us. 


\section{Appendix A: Quality of Service Parameters}

To obtain bounds on the queueing contribution to the QoS parameters when the traffic is passed through a single-server queue with constant service rate $s$, it is enough to have a linear bound on the logarithm of the queue-length distribution: if $-\mu$ represents the value of $\log \mathbb{P}(Q>1)$ then the straight line $-\delta q-\mu$ is an upper bound on the distribution $\log \mathbb{P}(Q>q)$ for all $q$, where

$-\delta=\lim _{q \rightarrow \infty} \frac{1}{q} \log \mathbb{P}(Q>q)$.

In a buffer of size $b$, the cell-loss ratio is given approximately by

$$
C L R(b) \approx \frac{\mathbb{P}(Q>b)}{\langle A\rangle} \leq \frac{e^{-\mu-\delta b}}{\langle A\rangle}
$$

where $\langle A\rangle$ denotes the mean activity of the traffic. To get bounds on the cell delay parameters, we can use the relation $D \approx Q / s$ between the delay $D$ experienced by a cell and the current queue-length $Q$ (the queue-length at the moment when the cell arrives at the buffer). For the mean cell-delay, we get

$$
\mathbb{E} D(b) \approx \frac{1}{s} \mathbb{E} Q(b)=\frac{1}{s} \sum_{q=0}^{b-1} \mathbb{P}(Q>q) \leq \frac{1}{s} \sum_{q=0}^{b-1} e^{-\mu-\delta b}
$$

and for the cell-delay variance,

$$
\begin{aligned}
\operatorname{var} D(b) & \approx \frac{1}{s^{2}} \sum_{q=0}^{b-1}(2 q+1) \mathbb{P}(Q>q)-\left(\frac{1}{s} \sum_{q=0}^{b-1} \mathbb{P}(Q>q)\right)^{2} \\
& \leq \frac{1}{s^{2}} \sum_{q=0}^{b-1}(2 q+1) e^{-\mu-\delta q}-\left(\frac{1}{s} \sum_{q=0}^{b-1} e^{-\mu-\delta q}\right)^{2}
\end{aligned}
$$

Proofs of these bounds will be given in Appendix B.

We see that the main problem in estimating the QoS parameters is that of estimating $\delta$; to explain how we do this, we have to go back to the idea of queue-length. The current queue-length $Q$ is the number of cells which have arrived less the number which have been served; to be precise, we have to make a couple of definitions:

- the number $A_{n}$ of cells which have arrived in the preceding $n$ clock-cycles is called the arrivals process;

- the work-load process $W_{n}$ is $A_{n}$ less the number of cells which could have been served in the preceding $n$ clock-cycles:

$W_{n}:=A_{n}-n s ;$ 
then a remarkable formula of queueing theory, see [1], gives

$Q=\max _{n \geq 0} W_{n}$.

Under very general conditions on the arrivals process, we know that $\log p(n, w)$, the $\log$ probability that $W_{n} / n$ exceeds the level $w$, is asymptotically linear in $n$ :

$\log p(n, w) \sim-n I(w)$.

(In the theory of large deviations, developed in recent years to estimate the probability of rare events, the coefficient $I$ is called the rate-function.) Denote by $f(q, s)$ the probability that the current queue-length $Q$ exceeds $q$ for given service-rate $s$; the theory of large deviations tells us [3] that

$\log f(b, s)$ is asymptotically linear in $b$ whenever $\log p(n, w)$ is asymptotically linear in $n$ :

$\log p(n, w) \sim-n I(w)$ implies $\log f(b, s) \sim-b \delta$

moreover, $\delta$ is determined by the rate-function $I$ :

$\delta=\min _{w>0} I(w) / w$

It follows that if we can determine $I$, then we can compute $-\delta$, the asymptotic slope of the graph of log-frequency of cell-loss versus buffer-size, and so estimate the QoS parameters.

\section{Appendix B: Proof of the Bounds}

Our starting point is the inequality $\mathbb{P}(Q>b) \leq e^{-\mu-\delta q}$. From this our bound on the cell loss ratio

$C L R(b) \approx \frac{\mathbb{P}(Q>b)}{\langle A\rangle} \leq \frac{e^{-\mu-\delta b}}{\langle A\rangle}$

follows immediately, and our bound on the mean cell delay

$\mathbb{E} D(b) \approx \frac{1}{s} \sum_{q=0}^{b-1} \mathbb{P}(Q>q) \leq \frac{1}{s} \sum_{q=0}^{b-1} e^{-\mu-\delta q}$

follows since every term in the sum is positive.

To justify our bound on the variance of the cell delay time we make use of the empirical observation that the ratio

$\frac{e^{-\mu-\delta q}}{\mathbb{P}(Q>q)}=: \alpha(q)$ 
is a non-decreasing function of $q$. To prove the bound we must prove that

$\operatorname{var} Q(b) \leq \frac{1}{s^{2}} \sum_{q=0}^{b-1}(2 q+1) e^{-\mu-\delta q}-\left(\frac{1}{s} \sum_{q=0}^{b-1} e^{-\mu-\delta q}\right)^{2}$

Let $F$ denote the right-hand side of the above inequality, and define $\nu$ and $\hat{\nu}$ by

$\nu=\sum_{q=0}^{b-1} \mathbb{P}(Q>q)=\mathbb{E} Q(b)$

$\hat{\nu}=\sum_{q=0}^{b-1} e^{\mu-\delta q} \geq \nu$

Then,

$$
\begin{aligned}
F-\operatorname{var} Q(b) & =\sum_{q=0}^{b-1}(2 q+1)\left(e^{-\mu-\delta q}-\mathbb{P}(Q>q)\right)+\nu^{2}-\hat{\nu}^{2} \\
& =\sum_{q=0}^{b-1}(2 q+1-\nu-\hat{\nu})\left(e^{-\mu-\delta q}-\mathbb{P}(Q>q)\right) \\
& \geq \sum_{q=0}^{b-1}(2 q+1-2 \hat{\nu})\left(e^{-\mu-\delta q}-\mathbb{P}(Q>q)\right)
\end{aligned}
$$

since $\hat{\nu} \geq \nu$. Rewriting this in terms of the function $\alpha(q)$ we get

$F-\operatorname{var} Q(b) \geq \sum_{q=0}^{b-1}(2 q+1-2 \hat{\nu}) \mathbb{P}(Q>q)(\alpha(q)-1)$

Those terms in the sum with $q<\hat{\nu}-1 / 2$ are negative, those with $q>\hat{\nu}-1 / 2$ are positive. Since $\alpha$ is a non-decreasing function of $q$, and $\alpha(q) \geq 1$ for all $q$, we therefore have

$$
\begin{aligned}
F-\operatorname{var} Q(b) & \geq(\alpha(\hat{\nu}-1 / 2)-1) \sum_{q=0}^{b-1}(2 q+1-2 \hat{\nu}) \mathbb{P}(Q>q) \\
& =(\alpha(\hat{\nu}-1 / 2)-1) \sum_{q=0}^{b}(q-\hat{\nu})^{2} \mathbb{P}(Q=q) \\
& \geq 0
\end{aligned}
$$

as required.

\section{Appendix C: Statistical Estimation Theory}

If $\lambda(\cdot)$ is the scaled cumulant generating function of the workload at a buffer of size $b$, then

$\lim _{b \rightarrow \infty} \frac{1}{b} \log \mathbb{P}[$ buffer overflow $]=-\delta$, 
where

$\delta=\sup \{\theta: \lambda(\theta) \leq 0)\}$.

Given a realisation of the workload process $\left\{X_{1}, \ldots, X_{n}\right\}$, we can form an estimate of the CGF $\lambda(\cdot)$ and hence of $\delta$. If the $X_{i}$ 's are IID, then the simplest estimate of the CGF is

$\hat{\lambda}_{n}(\theta)=\log \frac{1}{n} \sum_{i=1}^{n} e^{\theta X_{i}}$,

and the corresponding estimate of $\delta$ is

$\hat{\delta}_{n}=\sup \left\{\theta: \hat{\lambda}_{n}(\theta) \leq 0\right\}$.

$\hat{\delta}_{n}$ converges in probability to $\delta$, and so is a consistent estimator.

Since the $X_{i}$ 's are random variables, so are $\hat{\lambda}_{n}(\cdot)$ and $\hat{\delta}_{n}$, and we can construct a central limit theorem for the distribution of $\hat{\delta}_{n}$. We find it easier to work with $\phi(\cdot)$, the moment generating function, and we have that

$\hat{\delta}_{n}=\sup \left\{\theta: \hat{\phi}_{n}(\theta) \leq 1\right\}$,

where

$\hat{\phi}_{n}(\theta)=\frac{1}{n} \sum_{i=1}^{n} e^{\theta X_{i}}$

Now

$\mathbb{E}\left[\hat{\phi}_{n}(\theta)\right]=\frac{1}{n} \sum_{i=1}^{n} \mathbb{E}\left[e^{\theta X_{i}}\right]=\phi(\theta)$

and

$$
\begin{aligned}
\mathbb{E}\left[\hat{\phi}_{n}^{2}(\theta)\right] & =\frac{1}{n^{2}} \sum_{i=1}^{n} \sum_{j=1}^{n} \mathbb{E}\left[e^{\theta\left(X_{i}+X_{j}\right)}\right] \\
& =\frac{1}{n^{2}} \sum_{i=1}^{n} \mathbb{E}\left[e^{2 \theta X_{i}}\right]+\frac{1}{n^{2}} \sum_{i \neq j} \mathbb{E}\left[e^{\theta X_{i}} e^{\theta X_{j}}\right] \\
& =\frac{1}{n} \phi(2 \theta)+\frac{1}{n^{2}} \sum_{i \neq j} \mathbb{E}\left[e^{\theta X_{i}}\right] \mathbb{E}\left[e^{\theta X_{j}}\right] \\
& =\frac{1}{n} \phi(2 \theta)+\frac{n(n-1)}{n^{2}} \phi^{2}(\theta),
\end{aligned}
$$

and so

$\operatorname{Var}\left[\hat{\phi}_{n}(\theta)\right]=\frac{1}{n}\left(\phi(2 \theta)-\phi^{2}(\theta)\right)$. 
By the central limit theorem, if $\phi(2 \theta)<\infty$, then

$$
\frac{\sqrt{n}\left[\hat{\phi}_{n}(\theta)-\phi(\theta)\right]}{\sqrt{\phi(2 \theta)-\phi^{2}(\theta)}} \Rightarrow \chi,
$$

as $n \rightarrow \infty$, where $\Rightarrow$ denotes convergence in distribution and $\chi$ is a standard normal random variable. It follows that if $n$ is large, then

$$
\begin{aligned}
\mathbb{P}\left[\hat{\delta}_{n} \leq \theta\right] & =\mathbb{P}\left[\hat{\phi}_{n}(\theta) \geq 1\right] \\
& \approx \mathbb{P}\left[\chi \geq \frac{\sqrt{n}[1-\phi(\theta)]}{\sqrt{\phi(2 \theta)-\phi^{2}(\theta)}}\right] .
\end{aligned}
$$

Thus we can approximates the density $f_{n}(\theta)$ of the sampling distribution of $\hat{\theta}_{n}$ by

$$
\begin{aligned}
f_{n}(\theta) & =\frac{d}{d \theta} \mathbb{P}\left[\hat{\delta}_{n} \leq \theta\right] \\
& \approx \exp \left(\frac{-n[1-\phi(\theta)]^{2}}{\phi(2 \theta)-\phi^{2}(\theta)}\right) \frac{\sqrt{n}\left[(\phi(2 \theta)-\phi(\theta)) \phi^{\prime}(\theta)+(1-\phi(\theta)) \phi^{\prime}(2 \theta)\right]}{\sqrt{\phi(2 \theta)-\phi^{2}(\theta)}} .
\end{aligned}
$$

The distribution of $\hat{\delta}_{n}$ has an unfortunate feature: if $X_{i} \leq 0$ for all $i$, then $\hat{\phi}_{n}(\theta) \leq 1$ for all $\theta$ and so $\hat{\delta}_{n}=\infty$. Thus

$\mathbb{P}\left[\hat{\delta}_{n}\right]=\mathbb{P}\left[X_{1} \leq 0\right]^{n} \geq 0$,

so that $\mathbb{E}\left[\hat{\delta}_{n}\right]$ and all higher moments are infinite. However, if at least one of the $X_{i}$ 's is positive, then $\hat{\phi}_{n}(\theta)$ will be positive for $\theta$ sufficiently large. In general, it can be shown that if we condition on $k+1$ of the $X_{i}$ 's being positive, then the $k^{\text {th }}$ moment of the conditional distribution is finite.

Even if the $X_{i}$ 's are not IID, provided they are mixing, we can aggregate them into blocks of size $N$ large enough to ensure that the sequence $\left\{\tilde{X}_{k}\right\}_{k \geq 1}$, defined by

$\tilde{X}_{k}:=\sum_{i=(k-1) N+1}^{k N} X_{i}$,

is approximately IID.

\section{Results}

We investigated the properties of the estimator $\hat{\delta}_{n}$ empirically by applying it to simulations of some simple models. 


\section{IID Gaussian arrivals}

We simulated IID gaussian random variables with mean -0.25 and variance 1.0 , which corresponds to IID standard normal arrivals at the buffer being served at a constant rate 0.25 . We investigated how good the C.L.T. approximation was as a function of the length $n$ of the sequence of $X_{i}$ 's used to form the estimate $\hat{\delta}_{n}$. The same number, 10,000, of samples of $\hat{\delta}_{n}$ was used for each value of $n$.

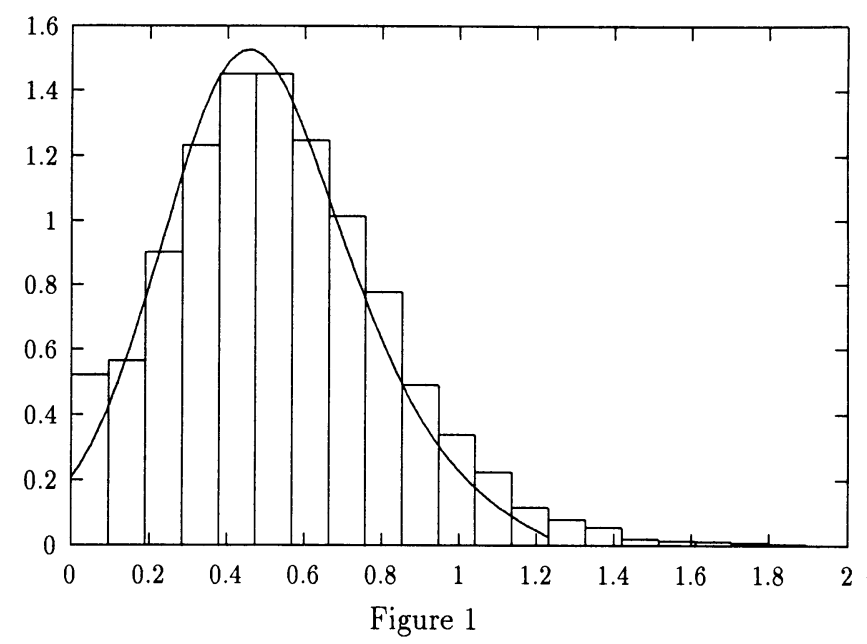

Fig. 1 shows the histogram of the empirical data plotted with the corresponding density calculated from the C.L.T. for $n=64$. The goodness of fit is only modest, and the most noticeable non-normal feature of the distribution is its skewness. Essentially the skewness is a result of the fact that, at this small value of $n$, the distribution has a high variance, but is bounded below by 0 , and so the high variance must come from mass distributed over the unbounded interval to the right. 


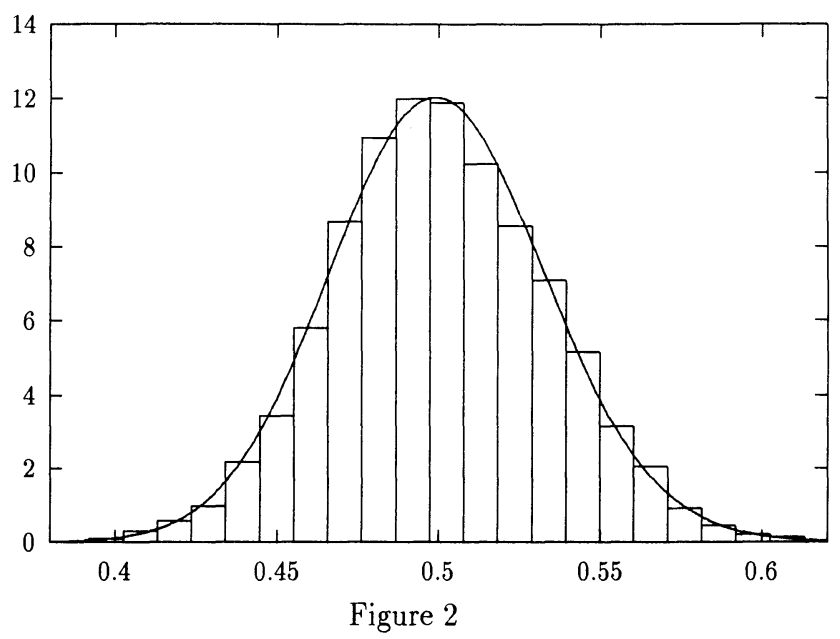

Fig.2 shows the empirical histogram and the C.L.T. density approximation for $n=$ 4096. As we expect, the fit is much better, and there is no noticeable skewness since the distribution is tightly and symmetrically centred on its mean.

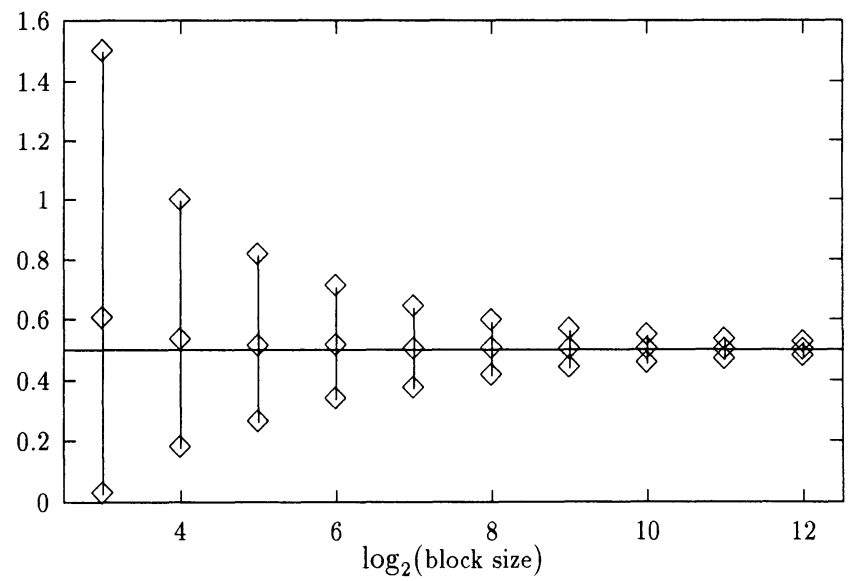

Figure 3

To illustrate how the variance and skewness of the sampling distribution of $\hat{\delta}_{n}$ decrease with increasing $n$, we plot the upper and lower quartiles and the median against $\log _{2} n$ for each of the empirical distributions in Fig.3. Note how the interquartile range becomes narrower and more symmetric about the median which is converging to the true value of $\delta$. 


\section{Correlated Gaussian arrivals}

If there are correlations between the $X_{i}$ 's, then our C.L.T. will no longer hold: the correlations tend to make the estimates of $\delta$ larger than the true value, so that $\hat{\delta}_{n}$ is no longer a consistent estimator. If, however, the $X_{i}$ 's are mixing, then we can aggregate them into blocks of size $N$ large enough to ensure that the sequence $\left\{\tilde{X}_{k}\right\}_{k \geq 1}$, defined by

$$
\tilde{X}_{k}:=\sum_{i=(k-1) N+1}^{\cdot k N} X_{i},
$$

is approximately IID. We can then base our estimate of $\delta$ on the $\tilde{X}_{i}$ 's, which will once more yield a consistent estimator. The larger we make $B$, the smaller the correlations in the aggregated process will be, and the better our estimate of $\delta$ will be. However, for a fixed length $n$ of the original sequence $\left\{X_{i}\right\}_{1 \leq i \leq n}$, the larger $B$ is the fewer (at most $\frac{n}{B}$ ) observations of the aggregated process we will have and thus the greater the variance of the estimate of $\delta$. This means that there is a trade-off between the consistency of $\hat{\delta}_{n}$ and its variance; we see from simulations that there is some optimal block-size to be chosen to form the estimate. We chose a model similar to the last one: we put $X_{i}=Z_{i}-0.25$ where $Z_{i}=0.5 Z_{i-1}+\chi_{i}$ and $\{\chi\}_{1 \leq i \leq n}$ is an IID sequence of standard normals. This corresponds to AR1 arrivals at the buffer being served at a constant rate 0.25 . We chose a value of 4096 for $n$, and examined how the sampling distribution of the estimator of $\delta$ based on the aggregated process varied with block- size. The same number, 10,000, of samples were taken in each case.

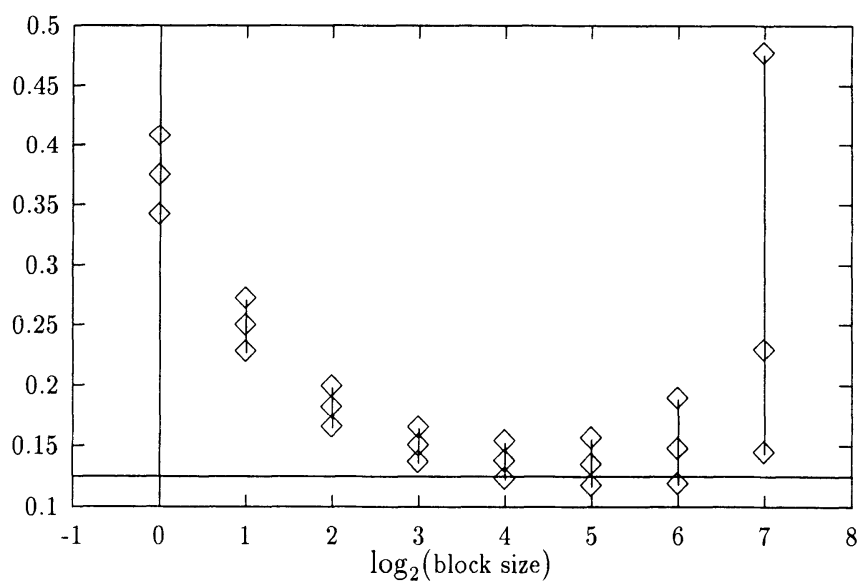

Figure 4

Fig.4 shows the upper and lower quartiles and the median of the empirical sampling distribution for each block-size used in the experiment. Note how, initially, the interquart- 
ile interval is far from the true value of $\delta$ but shifts down with increasing aggregation to contain $\delta$, only to explode out again at aggregation levels near the length of the original sequence.

\section{Corrections}

We have considered a number of first order corrections to this estimator. The first concerns the effect of correlations on our estimates of $\delta$ : if the sequence $\left\{X_{i}\right\}_{1 \leq i \leq n}$ is not IID but positively correlated then $\hat{\lambda}_{n}$ will tend to underestimate $\lambda$ and thus $\hat{\delta}_{n}$ will tend to overestimate $\delta$. The simplest first step towards compensating for this is to estimate the correlations and adjust $\hat{\lambda}_{n}$ accordingly: now

$$
\begin{aligned}
\lambda(\theta)= & \lim _{n \rightarrow \infty} \frac{1}{n} \log \mathbb{E}\left[e^{\theta \sum_{i=1}^{n}}\right] \\
= & \lim _{n \rightarrow \infty}\left(\frac{1}{n} \mathbb{E} \sum_{i=1}^{n} X_{i} \theta+\frac{1}{n}\left(\mathbb{E}\left(\sum_{i=1}^{n} X_{i}\right)^{2}-\left(\mathbb{E} \sum_{i=1}^{n} X_{i}\right)^{2}\right) \frac{\theta^{2}}{2}+\ldots\right) \\
= & \lim _{n \rightarrow \infty}\left(\frac{1}{n} \sum_{i=1}^{n} \mathbb{E} X_{i} \theta+\frac{1}{n} \sum_{i=1}^{n}\left(\mathbb{E} X_{i}^{2}-\left(\mathbb{E} X_{i}\right)^{2}\right) \frac{\theta^{2}}{2}+\right. \\
& \left.\frac{1}{n} \sum_{i<j}\left(\mathbb{E} X_{i} X_{j}-\mathbb{E} X_{i} \mathbb{E} X_{j}\right) \theta^{2}+\ldots\right) .
\end{aligned}
$$

This suggests that we should use

$\hat{\lambda}_{n}(\theta)=\log \frac{1}{n} \sum_{i=1}^{n} e^{\theta X_{i}}+\frac{1}{n} \sum_{i<j}\left(\mathbb{E} X_{i} X_{j}-\mathbb{E} X_{i} \mathbb{E} X_{j}\right) \theta^{2}$.

Note that if the sequence is positively correlated then the second term is positive, making our estimate

$\hat{\delta}_{n}=\sup \left\{\theta: \hat{\lambda}_{n}(\theta) \leq 0\right\}$

more conservative. In some sense, this correction is tailor-made for the case where the correlations decay exponentially; we suggest that the most fruitful application of these ideas is the case where we have aggregated our original data to the point where the correlations between blocks decay exponentially and apply this estimator. 


\section{References}

[1] A.A.Borovkov, Stochastic Processes in Queueing Theory, Springer, Berlin (1976)

[2] C.Courcoubetis, G.Kesidis, A.Ridder, J.Walrand and R.Weber, Admission control and routing in ATM networks using inferences from measured buffer occupancy, to appear in IEEE Trans. Comm.

[3] N.G.Duffield, N.O'Connell, Large Deviations and Overflow Probabilities for the General Single-Server Queue, with Applications DIAS Research Report DIASSTP-93-30 (1993)

[4] P.W.Glynn, W.Whitt, Logarithmic Asymptotics for Steady-State Tail Probabilities in a Single -Server Queue, to appear J.Appl.Prob. (1994)

[5] G.Kesidis, J.Walrand, C.S.Chang Effective Band-widths for Multiclass Markov Fluids and other ATM Sources, preprint (1993)

[6] O.E.Lanford, Entropy and equilibrium states in classical mechanics, in Statistical Mechanics and Mathematical Problems, A. Lenard, ed., Lecture Notes in Physics 20, Springer, Berlin (1973)

[7] J.T.Lewis, C.-E.Pfister, Thermodynamic Probability Theory: some aspects of large deviations, preprint DIAS-93-33 (1993), to appear in Theor.Prob.Appl.

[8] D.Ruelle, Correlation functionals, J.Math.Phys. 6: 201 (1965)

[9] D.Ruelle, Chance and Chaos, Princeton U.P., Princeton (1991)

[10] G.de Veciana, C.Courcoubetis and J.Walrand, Decoupling bandwidths for networks: a decomposition approach to resource management, Memorandum \# UCL/ERL M93/50, University of California 